\title{
Decreased expression of carbonyl reductase 1 promotes ovarian cancer growth and proliferation
}

\author{
YUKI OSAWA, YOSHIHITO YOKOYAMA, TATSUHIKO SHIGETO, \\ MASAYUKI FUTAGAMI and HIDEKI MIZUNUMA
}

Department of Obstetrics and Gynecology, Hirosaki University

Graduate School of Medicine, Hirosaki, Aomori 036-8562, Japan

Received October 21, 2014; Accepted December 3, 2014

DOI: $10.3892 / \mathrm{ijo} .2014 .2810$

\begin{abstract}
Carbonyl reductase 1 (CBR1) expression level is related to tumor progression. Decreased CBR1 expression is associated with poor prognosis in ovarian cancer. We investigated the relationship between CBR1 expression level and malignant potential of ovarian cancer. OVCAR-3 cells overexpressing CBR1 or knocked down for CBR1 were obtained by transfecting CBR1 plasmid DNA (pDNA) or small interfering RNA (siRNA) by electroporation. In vitro cell proliferation and invasion were compared between the two cell types. Subcutaneous CBR1-overexpressed OVCAR-3 cells $(n=10)$ and wild-type ones $(n=5)$ were injected into nude mice. The CBR1 siRNA was then injected twice a week into five of the 10 CBR1-overexpressed OVCAR-3 tumors. Tumor growth and metastatic behavior were observed 3 weeks after cell transplantation. Cell proliferation significantly decreased in CBR1-overexpressed cells as compared to the control, whereas cell proliferation and invasion significantly increased in CBR1-suppressed cells as compared to the control. The size of the CBR1 siRNA-injected tumors $(n=5)$ increased significantly as compared to the other two groups ( $n=5$ for each group). The number of metastatic foci in the lungs was significantly higher in mice injected with CBR1 siRNA (7.0 \pm 2.0$)$ compared to CBR1-overexpressed

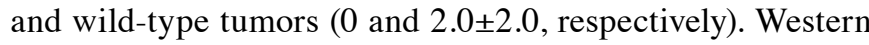
blot analysis showed that, while vascular endothelial growth factor (VEGF)-C expression was stable in the CBR1-siRNA-injected tumors, E-cadherin expression was decreased, whereas matrix metalloproteinase (MMP)-9 was increased in CBR1-siRNA-injected tumors compared to the other two groups. These results showed that CBR1 decreases promoted tumor proliferation and growth as well as invasion
\end{abstract}

Correspondence to: Dr Yoshihito Yokoyama, Department of Obstetrics and Gynecology, Hirosaki University Graduate School of Medicine, 5-Zaifu-cho, Hirosaki, Aomori 036-8562, Japan

E-mail: yokoyama@cc.hirosaki-u.ac.jp

Key words: carbonyl reductase 1, ovarian cancer, siRNA, MMP-9, E-cadherin, VEGF-C and metastasis, suggesting that CBR1 has potential to become a new candidate for molecular targeting therapy.

\section{Introduction}

Epithelial ovarian cancer (EOC) is the world's most lethal gynecological cancer, and the World Health Organization Global Database listed EOC as the 7th leading form of cancer in women in 2008 (1).

Although the mean 5-year survival rate for EOC has improved significantly over the past 30 years, the prognosis remains poor, with a $46 \% 5$-year survival rate (2). The prognosis for EOC is closely related to the cancer clinical stage at diagnosis. The mean 5-year survival rate in advanced stages (FIGO stage III or IV) is as low as $11-41 \%$ (2). More than $70 \%$ of EOCs are detected in the advanced stages mainly because of a lack of early warning signs and reliable diagnostic tests. Cytoreductive surgery followed by adjuvant chemotherapy is recommended as the primary treatment for advanced EOC. Postoperatively, a taxane and carboplatin combination is used as the first-line chemotherapy. EOC is highly responsive to initial anticancer treatment, but approximately half of the advanced cases recur within 2 years and result in poor prognosis due to a decreased response to chemotherapy (3). Therefore, new clinically useful biomarkers and new targets for EOC treatment need to be identified in order to initiate intensive treatment.

Carbonyl reductase 1 (CBR1) is an NADPH-dependent oxidoreductase with broad specificity for carbonyl compounds, which reduces aldehydes and ketones (4). CBR1 has been isolated from various organs such as the liver, kidney, breast, ovary, and vascular endothelial cells (5), and has been studied for its function in the metabolism of a variety of drugs such as anthracycline, daunorubicin, haloperidol, and doxorubicin $(6,7)$. Another important CBR1 function is to convert prostaglandin (PG) $\mathrm{E}_{2}$ to $\mathrm{PGF}_{2 \alpha}(8,9)$. $\mathrm{PGE}_{2}$ have been demonstrated not only to modulate apoptosis and $\mathrm{Bcl}-2$ expression (10), but also to induce angiogenesis (11).

CBR1 has been reported to relate to tumor progression (12-14). Suppression of CBR1 expression was associated with poor prognosis in uterine endometrial cancer and uterine cervical squamous cell carcinoma $(12,13)$. Our previous studies showed that decreased CBR1 expression is associated 
with lymph node metastasis and poor prognosis in ovarian cancer (14), and induction of CBR1 expression in ovarian tumors leads to a spontaneous decrease in tumor size (15).

In vitro experiments showed that $\mathrm{CBR} 1$ suppression enhanced uterine squamous cell carcinoma and endometrial carcinoma malignant behavior $(12,13)$. A significant inverse relationship between vascular endothelial growth factor (VEGF) and CBR1 expression was observed in cancer tissues (16). The epithelial mesenchymal transition (EMT) has been associated with tumor progression and poor prognosis in various human cancers. Predominantly, the functional loss of E-cadherin in epithelial cells is a common feature of EMT (17). Earlier studies showed that CBR1 is likely to be associated with EMT in endometrial adenocarcinoma (13). Furthermore, the extracellular matrix (ECM) degradation is necessary for cancer cells to invade. Matrix metalloproteinases (MMPs) are enzymes that resolve ECM. Loss of E-cadherin and activation of MMP-9 have been reported to correlate with poor prognosis in ovarian cancer (18). Because CBR1 molecular mechanisms remain unclear in ovarian cancer, it may be of interest to investigate the relationship between altered expression of CBR1 and molecules such as VEGF, E-cadherin, and MMPs that affect malignant behavior.

In this study, we investigated the effect of decreased CBR1 expression on proliferation of ovarian cancer cells and growth of ovarian cancer, and aimed to elucidate its mechanisms of action.

\section{Materials and methods}

Cell line and culture. OVCAR-3 was obtained from the American Type Culture Collection (Rockville, MD, USA). OVCAR-3 cells have been commonly used to produce xenografted solid tumor (16) and are derived from human ovarian cancer. They were cultured in RPMI-1640 medium supplemented with $10 \%$ fetal calf serum (FCS), $100 \mathrm{U} / \mathrm{ml}$ penicillin and $100 \mathrm{mg} / \mathrm{ml}$ streptomycin at $37^{\circ} \mathrm{C}$ in a water-saturated atmosphere with $5 \% \mathrm{CO}_{2} / 95 \%$ air.

Animal experiments. Animal experiments were conducted in accordance with the Guidelines for Animal Experimentation, Hirosaki University (Aomori, Japan). Eight-week-old female $\mathrm{BALB} / \mathrm{c} \mathrm{nu} / \mathrm{nu}$ mice were used in this study. All mice were group housed in plastic cages with stainless steel grid tops in an air-conditioned and 12-h light/dark cycle-maintained room in the Institute for Animal Experiments of Hirosaki University and fed with water and food ad libitum.

Plasmid DNA preparation. To optimize and obtain highly efficient transfection, we used a pCMV6-AC-GFP vector (OriGene Technologies, Inc., Rockville, MD, USA) that encodes the human CBR1, GFP and ampicillin-resistant gene. For amplification, pCMV6-AC-GFP was transformed into E. coli-DH5 $\alpha$ competent cells by heat shock transformation according to standard laboratory protocols. The transformed bacteria were amplified in LB-ampicillin medium. The plasmids were purified from cultured transformed bacteria using a PureLink HiPure Plasmid Filter Miniprep DNA purification kit (Invitrogen Life Technologies, Carlsbad, CA, USA) according to the manufacturer's protocol. Plasmid DNA (pDNA) was diluted in sterile water at a concentration of $3 \mu \mathrm{g} / \mu \mathrm{l}$.

Small interfering RNA preparation. The sequences of small interfering RNA (siRNA) duplexes specific to CBR1 were synthesized commercially by Invitrogen Life Technologies. CBR1 siRNA sense, 5'-AUACGUUCACCACUCUCCCTT-3' and antisense, 5'-GGGAGAGUGGUGAACGUAUTT-3' were designed to target different coding regions of the human CBR1 mRNA sequence (GeneBank Accession no. NM_001757).

Transfection. OVCAR-3 cells were trypsinized at a density of $5.0 \times 10^{5}$ cells/plate and were rinsed twice with serum-free RPMI-1640. The cells were then transferred into an electroporation cuvette. Afterwards, $10 \mu \mathrm{l}$ of human CBR1 pDNA was added to the cells and electroporated with the square wave program with poring pulses and transfer pulses using a NEPA21 (Nepa Gene Co., Ltd., Chiba, Japan). Electroporated OVCAR-3 cells were then immediately transferred to the culture plates containing RPMI-1640 with fetal bovine serum (FBS). Transfected cells were harvested 4 days later. The control was only given electric stimulation by electroporation.

Cell proliferation. OVCAR-3 cells were cultured in 6-well plates as described in the cell line and culture paragraph above. Cell counts were performed $24 \mathrm{~h}$ in a logarithmic growth phase after transfection of OVCAR-3 cells with CBR1-DNA or CBR1-siRNA. To distinguish live and dead cells, cells were stained with $0.3 \%$ trypan blue solution (Wako Pure Chemicals Industries, Ltd., Osaka, Japan). Cells were counted using a hemocytometer. The cell count was performed in triplicate and the total cell counts are presented as averages.

Invasion assay. The tumor cell invasiveness was determined using the CytoSelect 24-Well Cell Invasion Assay kit (basement membrane, colorimetric format; Cell Biolabs, Inc., San Diego, CA, USA) according to the manufacturer's instructions. For invasion assays, the cells were cultured in serum-free culture medium at a density of $1.0 \times 10^{5}$ cells/well in the upper chamber, which had an $8.0-\mu \mathrm{m}$ pore size membrane coated with a uniform layer of basement membrane matrix solution, and the lower chamber was filled with the culture medium with $10 \%$ FBS. After incubation for $48 \mathrm{~h}$, invasive cells on the bottom of the membrane were stained with Diff-Quick (Sysmex Corp., Hyōgo, Japan) and quantified using an absorption photometer $(\mathrm{OD}=560 \mathrm{~mm})$.

Xenograft mouse model. The mice were divided into three groups ( $\mathrm{n}=5$ for each group). OVCAR-3 cells or OVCAR-3 expressing the CBR1-DNA $\left(5.0 \times 10^{5}\right.$ cells $)$ were inoculated subcutaneously in $0.2 \mathrm{ml}$ of RPMI-1640 medium in the back region of nude mice. All the mice were numbered, housed separately, and tumor development was examined for 10 days. The examination started once the longer diameter of the tumor reached $5 \mathrm{~mm}$ (day 0). The control group and the CBR1-DNA group were intratumorally administered $5 \%$ glucose solution, while the CBR1-siRNA group was intratumorally injected with the CBR1-siRNA using Invivofectamine (Invitrogen Life Technologies) at a dose of $80 \mu \mathrm{g}$ twice a week on day 0 and 7. Mice were monitored every day for tumor growth until 


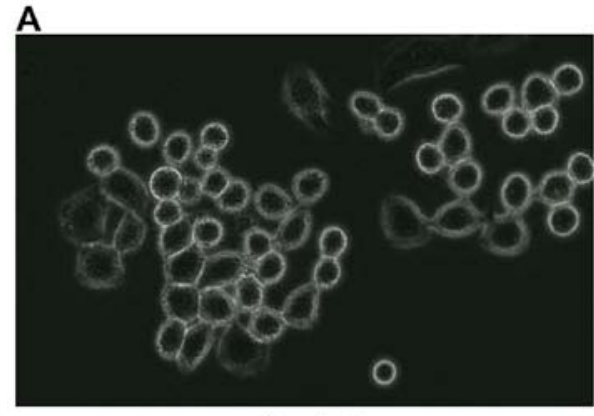

Control

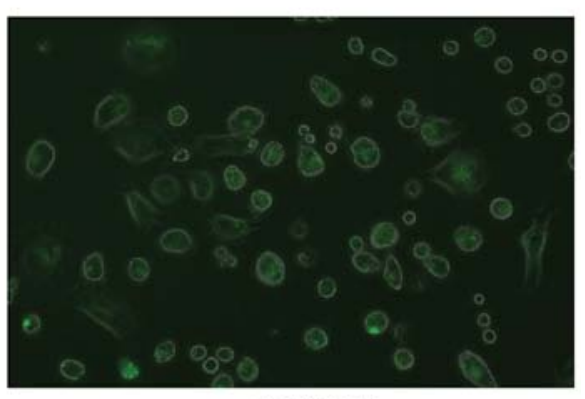

CBR1-GFP

B

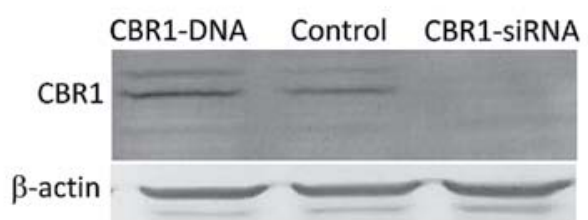

Figure 1. Transfection of carbonyl reductase 1 (CBR1) DNA and CBR1 small interfering RNA (siRNA) into OVCAR-3 cells. (A) Fluorescent photographs (overlay with the phase difference) of OVCAR-3 cells transfected with pCMV6-AC-GFP-expressing CBR1 taken $48 \mathrm{~h}$ after transfection. (B) CBR1 expression levels measured by western blot analysis. $\beta$-actin was used as an internal control.

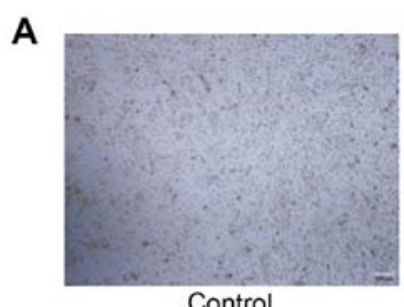

Control

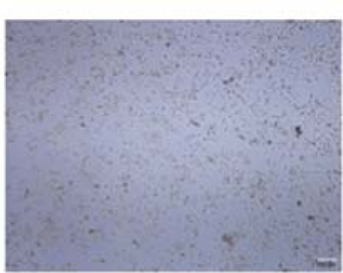

CBR1-DNA

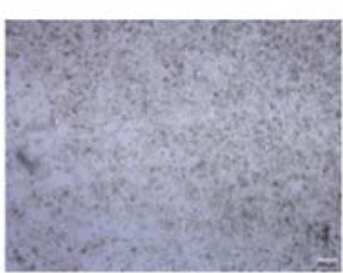

CBR1-SiRNA

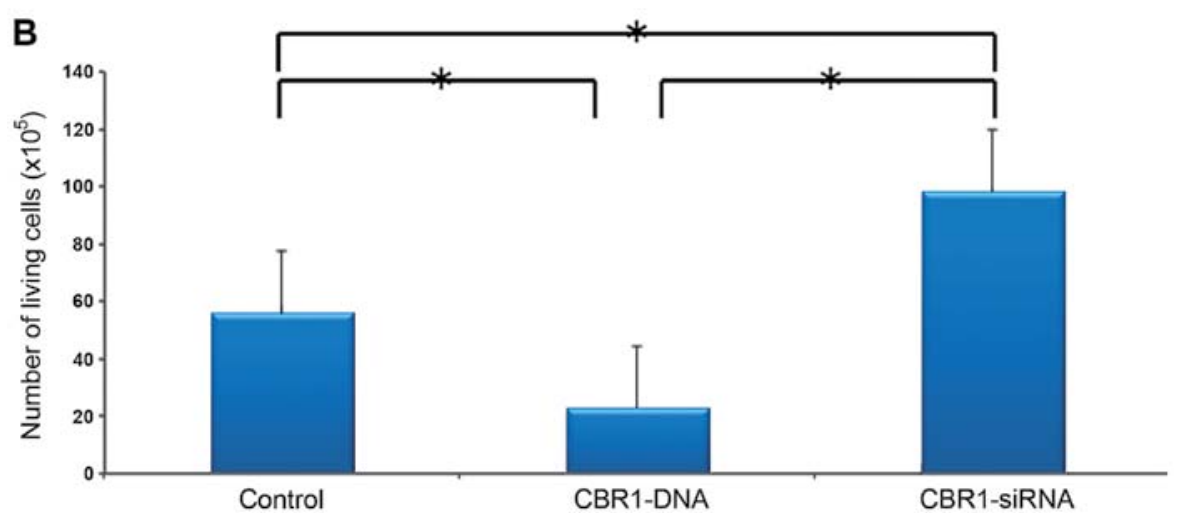

Figure 2. Cell proliferation. (A) Cell density among the three groups. Carbonyl reductase 1 (CBR1)-small interfering RNA (siRNA)-tranfected cells grew in multilayers. Magnification, x20. (B) The number of living cells was significantly higher in CBR1-siRNA group than in the other two groups and significantly lower in CBR1-DNA group than in the control group. Results are shown as the mean \pm standard deviation. ${ }^{*} \mathrm{P}<0.001$.

day 14. The tumor dimensions were measured every day using caliper and tumor volume was calculated using the equation: $\mathrm{V}\left(\mathrm{mm}^{3}\right)=\mathrm{A} \mathrm{x} \mathrm{B} / 2$, where $\mathrm{A}$ is the largest diameter and $\mathrm{B}$ is the smallest diameter (19). The mice were sacrificed on day 14 to remove the tumor and lungs for pathologic and biochemical studies.

Western blot analysis. Cell lysates (50 $\mu \mathrm{g}$ protein) were prepared from tumor tissues, electrophoresed through a $12.5 \%$ sodium dodecyl sulfate polyacrylamide gel, and blotted as described previously (16). The protein concentration was determined using Bradford's method. The blots were probed with the following diluted antibodies for $2 \mathrm{~h}$ : CBR1 at 1:200, human VEGF-C at 1:200 (both from Santa Cruz Biotechnology, Inc., Santa Cruz, CA, USA), E-cadherin at 1:100,000 (GeneTex, Inc., Irvine, CA, USA), MMP-9 at 1:500 (Abnova, Walnut, CA, USA), and $\beta$-actin at 1:2,000 (Sigma-Aldrich, St. Louis, MO, USA). The membranes were then incubated for $1 \mathrm{~h}$ with the appropriate biotinylated secondary antibodies, transferred to avidin-biotin-peroxidase complex reagent, and incubated 


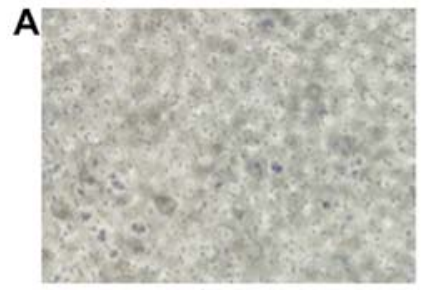

Control

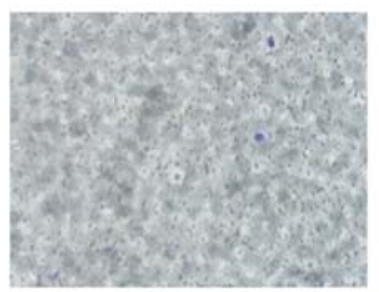

CBR1-DNA

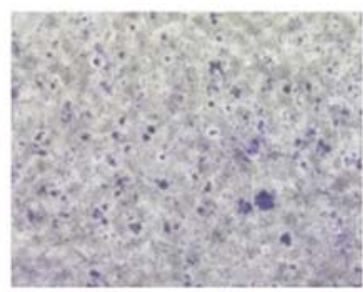

CBR1-siRNA

B

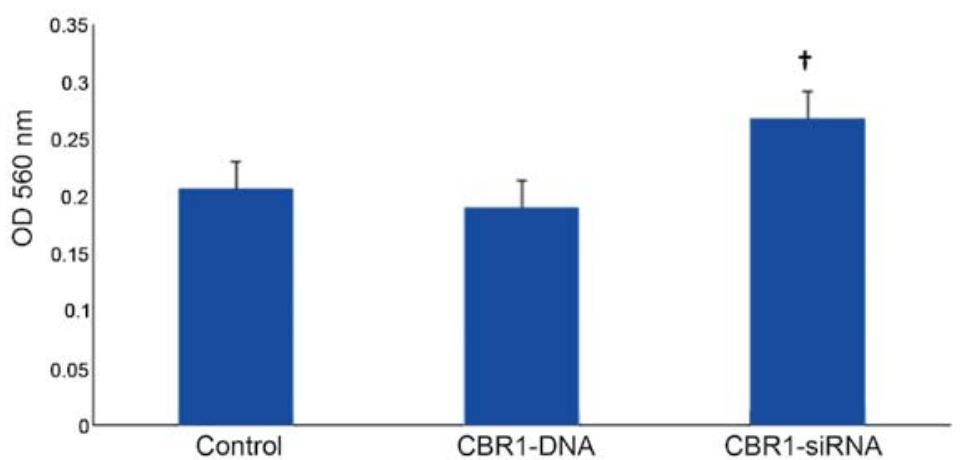

Figure 3. Cell invasion. (A) Cell invasion $48 \mathrm{~h}$ after transfection. Invasive cells were stained in blue. Magnification, $\mathrm{x} 100$. (B) Cell invasion activity was significantly higher in carbonyl reductase 1 (CBR1)-small interfering RNA (siRNA)-transfected cells than in both the control and CBR1-DNA-transfected cells. ${ }^{\dagger} \mathrm{P}<0.05$ versus control and CBR1-DNA.

in this solution for $30 \mathrm{~min}$. Diaminobenzidine was used as a substrate.

Statistical analysis. The Tukey-Kramer test was used to assess differences in the number of living cells between the control, CBR1-DNA, and CBR1-siRNA group. Differences in the invasion assay between the three groups were evaluated using Student's t-test. Differences in tumor volume between the control, CBR1-DNA, and CBR1-siRNA group were evaluated using the Mann-Whiney $\mathrm{U}$ test. A probability value of $\mathrm{P}<0.05$ was considered to be significant.

\section{Results}

Comparison of CBR1 expression levels. CBR1 expression levels were compared in OVCAR-3 cells transfected with CBR1-DNA or CBR1-siRNA. CBR1-GFP protein fluorescence was clearly detected in CBR1-DNA-tranfected cells (Fig. 1A). Western blot analysis showed that CBR1 expression level was higher in CBR1-DNA-transfected cells and lower in CBR1-siRNA-tranfected cells when compared to the control cells (Fig. 1B).

Cell proliferation. Difference in cell density was demonstrated among the three groups as shown in Fig. 2A. The CBR1-siRNA-tranfected cells grew in multilayers. Cell density was lower in CBR1-DNA-transfected cells and higher in CBR1-siRNA-transfected cells when compared to the control cells (Fig. 2A). The cell counts were $55.6 \times 10^{5}$ cells for the control group, $22.8 \times 10^{5}$ cells for the CBR1-DNA group, and $98.1 \times 10^{5}$ cells for the CBR1-siRNA group, respectively. The number of living cells was significantly higher in the CBR1-siRNA group than in the other two groups and significantly lower in CBR1-DNA group than in the control group (Fig. 2B, $\mathrm{P}<0.001$ each).
Cell invasion. Fig. 3A indicates the appearance of invasive cells $48 \mathrm{~h}$ after transfection. Invasive cells were stained in blue. Cell invasion was significantly higher in CBR1-siRNA-transfected cells than in both the control and the CBR1-DNA-transfected cells (Fig. 3B, $\mathrm{P}<0.05$ ).

Tumor growth. In the xenograft mouse model, tumor volumes were significantly reduced from the first day after initial infection with the CBR1-siRNA in the CBR1-siRNA group $(n=5)$ compared to the control $(n=5)$ and CBR1-DNA group $(\mathrm{n}=5)$ (Fig. 4, $\mathrm{P}<0.05)$. The same trend lasted until they were sacrificed at day 14. Although tumor growth in the CBR1-DNA group was suppressed compared to the control group, there was no significant difference between the two groups. Inset photos are representatives of each group at day 14 .

Tumor invasion and metastasis. The mice were sacrificed on day 14 to remove the tumor and lungs and to observe the abdominal cavity. The number of metastatic foci in the lungs was $7.0 \pm 2.0,0$, and $2.0 \pm 2.0$ in mice bearing CBR1-siRNA-injected, CBR1-DNA, and the control tumors, respectively. Metastatic foci to the lungs were significantly increased in CBR1-siRNA group compared with the other two groups (Fig. 5, $\mathrm{P}<0.05$ ). There was no metastatic lesion in the lungs of CBR1-DNA tumor-bearing mice. Most of the mice in the CBR1-siRNA group presented a deep invasion of subcutaneous tumors into the abdominal cavity (data not shown).

Altered expression of VEGF-C, E-cadherin, and MMP-9 in the tumors according to CBR1 expression levels. Western blot analysis showed that, while VEGF-C expression was decreased in the CBR1-DNA tumors and was stable in the CBR1-siRNA tumors, E-cadherin-decreased expression and MMP-9-increased expression were observed in the CBR1-siRNA tumors compared to the other two groups (Fig. 6). 


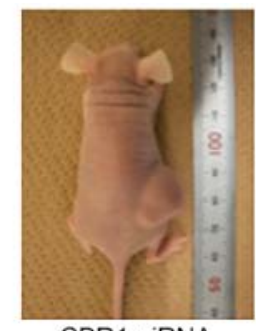

CBR1-siRNA

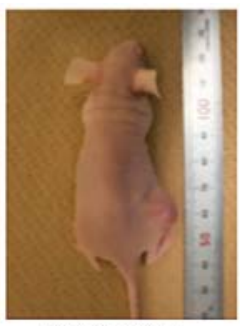

CBR1-DNA

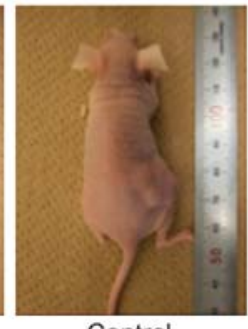

Control

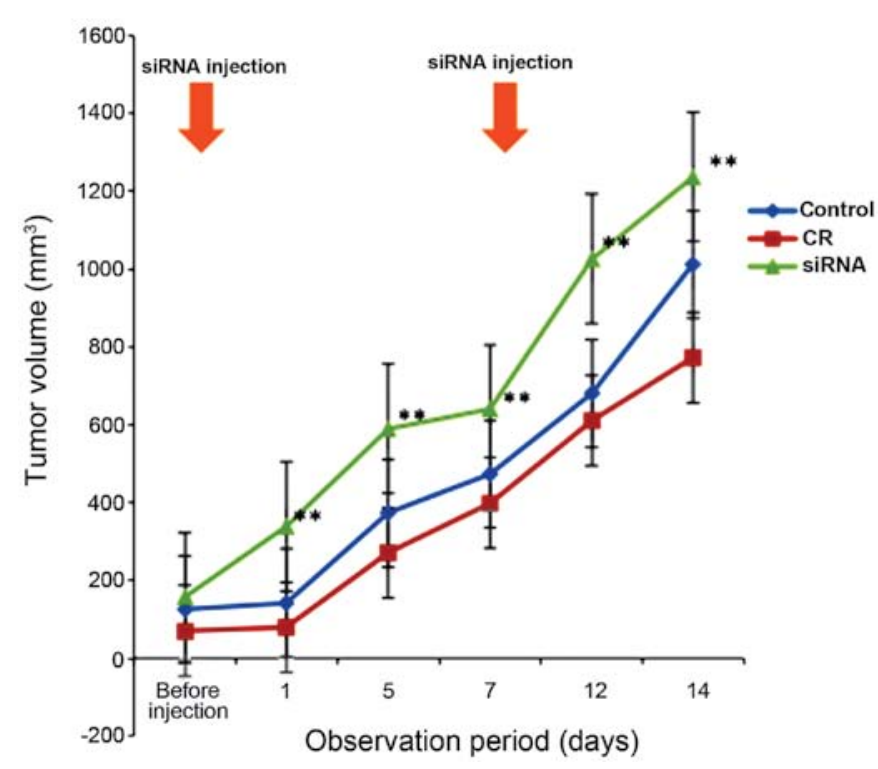

Figure 4. Comparison of tumor growth in a xenograft mouse model. Tumor volumes were significantly reduced from the first day after initial infection with the carbonyl reductase 1 (CBR1)-small interfering RNA (siRNA) in the CBR1-siRNA group $(n=5)$ compared with the control $(n=5)$ and CBR1-DNA group $(n=5)$. Results are shown as the mean \pm standard deviation. Inset photographs are representative of each group during the second week of treatment. ${ }^{* *} \mathrm{P}<0.05$ versus the control and CBR1-DNA group.

\section{Discussion}

In this study using ovarian cancer cells, CBR1 suppression by siRNA transfection showed a significantly higher proliferative ability and invasive activity that caused rapid tumor growth and lung metastasis than the control cells. In addition, CBR1 suppression led to decreased expression of E-cadherin and increased expression of MMP-9 in tumors, whereas VEGF-C expression remained stable. Results of the present study were consistent with earlier findings obtained in clinical (12-14) and animal studies (20). Umemoto et al reported that CBR1 loss or decrease was significantly related to retroperitoneal lymph node metastasis and poor outcome in EOC (14). Murakami et al showed a significantly close relationship between decreased CBR1 expression and progression-free survival as well as overall survival in uterine cervical or endometrial cancer $(12,13)$. Ismail et al, on the other hand, showed that mouse cancer cells in which CBR1 was knocked down by transfection of an antisense CBR1 cDNA acquired a potent metastatic potential (20). In addition, our previous study showed that ovarian tumors derived from CBR1 sense cDNA-transfected cells grew up to the second week, but then decreased continuously until the fifth week of observation (15). We have shown that the spontaneous regression was due to
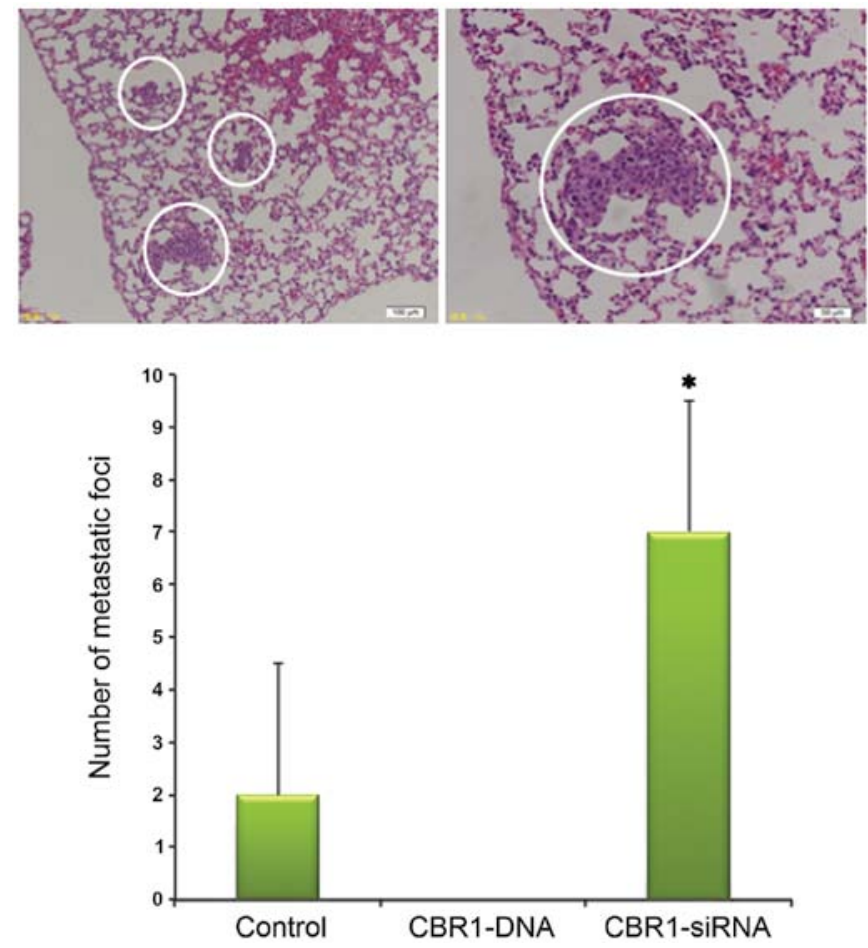

Figure 5. Comparison of lung metastasis among the three groups. Metastatic foci to the lungs were significantly increased in the carbonyl reductase 1 (CBR1)-small interfering RNA (siRNA) group compared with the other two groups. Inset photographs represent lung micrometastasis in mice given CBR1-siRNA observed during the second week of treatment. Lung metastatic foci are highlighted in white circles. ${ }^{*} \mathrm{P}<0.05$ versus the control and CBR1-DNA group.

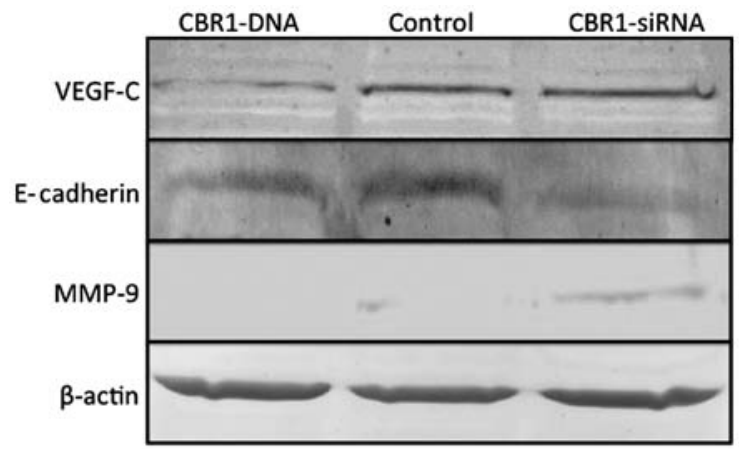

Figure 6. Vascular endothelial growth factor (VEGF)-C, E-cadherin, and matrix metalloproteinase (MMP)-9 expression in tumors is altered based on carbonyl reductase 1 (CBR1) expression levels. VEGF-C expression was decreased in CBR1-DNA tumors and was stable in CBR1-small interfering RNA (siRNA) tumors. CBR1-siRNA tumors presented decreased E-cadherin expression and increased MMP-9 expression compared to the other two groups.

increased necrosis through phagocytosis of apoptotic cells by phagocytes attracted by increased eat-me-signal induced by CBR1 (15). Furthermore, CBR1 expression vector transfection into mouse ovarian cancer cells induced a significant reduction of $\mathrm{PGE}_{2}$ level and VEGF expression (16). These findings confirm that CBR1 expression is involved in cancer cell growth and strongly indicate that CBR1 expression influences cancer cell acquisition of malignant and metastatic potential.

In this study, CBR1 suppression in ovarian cancer induced a decrease in E-cadherin expression and an increase in MMP-9 
expression. The loss of E-cadherin is known as a marker of EMT that is associated with carcinoma progression and poor prognosis in malignant tumors (21). Because MMPs resolve ECM, the MMP increase is necessary to acquire a potent invasive potential. Among MMP subtypes, MMP-9 is closely associated with poor outcome in ovarian cancer (22) and its expression level is significantly stronger as lesions progressed from a benign tumor to advanced carcinoma $(23,24)$. Earlier study showed that MMP-9 overexpression led to a loss of E-cadherin and promoted a migratory and invasive phenotype in ovarian cancer cells (25), supporting the present results. On the other hand, in this study, although VEGF-C expression was decreased in the CBR1-DNA tumors, its expression in CBR1-siRNA tumors was similar to that of the control ovarian cancer cell tumors. VEGF subtypes are commonly known to be involved in cancer metastasis (26). VEGF receptor (VEGFR)-3, a tyrosine kinase receptor, is involved in lymphangiogenesis and distant metastasis. VEGF-C is a specific ligand of VEGFR-3 (27). Stable expression of VEGF-C/VEGFR-3 increased distant metastasis, including lungs and lymph node metastasis in various types of malignant tumor (28-31). The present study showed that lung metastasis was significantly more frequent in CBR1-siRNA tumors than in the other two groups. Additionally, lung metastasis did not occur in CBR1-DNA tumors. The present results suggest that the increase in MMP-9 and decrease in E-cadherin induced by reducing CBR1 expression may enhance malignant behavior such as invasion and metastasis in ovarian cancer under stable expression of VEGF-C.

CBR1 exists in various tissues in humans (5). It is important to confirm whether CBR1 actually functions as an enzyme in cancer cells. Our previous study showed that $\mathrm{PGE}_{2}$ levels were reduced in OVCAR-3 cells transfected with CBR1 sense cDNA (16) and that the increase of E-cadherin expression was blocked by quercetin, which inhibits CBR1 enzymatic activities (12), suggesting that the effects of CBR1 are due to its enzymatic activities rather than its structural effects.

In conclusion, it emerged that CBR1 loss or decrease promoted tumor proliferation and growth as well as invasion and metastasis in this study, suggesting that CBR1 might become a new candidate for molecular targeting therapy.

\section{Acknowledgements}

This study was supported by a Grant-in-Aid for Cancer Research from the Ministry of Education, Culture, Sports, Science and Technology (Tokyo, Japan) (no. 20591935 to Dr Y. Yokoyama).

\section{References}

1. Siegel R, Naishadham D and Jemal A: Cancer statistics, 2013. CA Cancer J Clin 63: 11-30, 2013.

2. Heintz AP, Odicino F, Maisonneuve P, et al: Carcinoma of the ovary. FIGO 26th Annual Report on the Results of Treatment in Gynecological Cancer. Int J Gynaecol Obstet 95 (Suppl 1): S161-S192, 2006.

3. Yokoyama Y, Futagami M, Watanabe J, et al: Redistribution of resistance and sensitivity to platinum during the observation period following treatment of epithelial ovarian cancer. Mol Clin Oncol 2: 212-218, 2014.
4. Gonzalez-Covarrubias V, Ghosh D, Lakhman SS, Pendyala L and Blanco JG: A functional genetic polymorphism on human carbonyl reductase 1 (CBR1 V88I) impacts on catalytic activity and NADPH binding affinity. Drug Metab Dispos 35: 973-980, 2007.

5. Wermuth B, Bohren KM, Heinemann G, von Warthburg JP and Gabbay KH: Human carbonyl reductase. Nucleotide sequence analysis of a cDNA and amino acid sequence of the encoded protein. J Biol Chem 263: 16185-16188, 1988

6. Plebuch M, Soldan M, Hungerer C, Koch L and Maser E: Increased resistance of tumor cells to daunorubicin after transfection of cDNAs coding for anthracycline inactivating enzymes. Cancer Lett 255: 49-56, 2007.

7. Olson LE, Bedja D, Alvey SJ, Cardounel AJ, Gabrielson KL and Reeves RH: Protection from doxorubicin-induced cardiac toxicity in mice with a null allele of carbonyl reductase 1. Cancer Res 63: 6602-6606, 2003.

8. Schieber A, Frank RW and Ghisla S: Purification and properties of prostaglandin 9-ketoreductase from pig and human kidney. Identity with human carbonyl reductase. Eur J Biochem 206: 491-502, 1992.

9. Waclawik A and Ziecik AJ: Differential expression of prostaglandin (PG) synthesis enzymes in conceptus during peri-implantation period and endometrial expression of carbonyl reductase/PG 9-ketoreductase in the pig. J Endocrinol 194: 499-510, 2007.

10. Sheng H, Shao J, Morrow JD, Beauchamp RD and DuBois $\mathrm{RN}$. Modulation of apoptosis and $\mathrm{Bcl}-2$ expression by prostaglandin E2 in human colon cancer cells. Cancer Res 58: 362-366, 1998.

11. Tsujii M, Kawano S, Tsuji S, Sawaoka H, Hori M and DuBois RN: Cyclooxygenase regulates angiogenesis induced by colon cancer cells. Cell 93: 705-716, 1998.

12. Murakami A, Fukushima C, Yoshidomi K, et al: Suppression of carbonyl reductase expression enhances malignant behaviour in uterine cervical squamous cell carcinoma: carbonyl reductase predicts prognosis and lymph node metastasis. Cancer Lett 311: 77-84, 2011.

13. Murakami A, Yakabe K, Yoshidomi K, et al: Decreased carbonyl reductase 1 expression promotes malignant behaviours by induction of epithelial mesenchymal transition and its clinical significance. Cancer Lett 323: 69-76, 2012.

14. Umemoto M, Yokoyama Y, Sato S, Tsuchida S, Al-Mulla F and Saito Y: Carbonyl reductase as a significant predictor of survival and lymph node metastasis in epithelial ovarian cancer. Br J Cancer 85: 1032-1036, 2001.

15. Wang H, Yokoyama Y, Tsuchida S and Mizunuma H: Malignant ovarian tumors with induced expression of carbonyl reductase show spontaneous regression. Clin Med Insights Oncol 6: 107-115, 2012.

16. Yokoyama Y, Xin B, Shigeto T, et al: Clofibric acid, a peroxisome proliferator-activated receptor alpha ligand, inhibits growth of human ovarian cancer. Mol Cancer Ther 6: 1379-1386, 2007.

17. Yang $\mathbf{J}$ and Weinberg RA: Epithelial-mesenchymal transition: at the crossroads of development and tumor metastasis. Dev Cell 14: 818-829, 2008.

18. Alshenawy HA: Immunohistochemical expression of epidermal growth factor receptor, E-cadherin, and matrix metalloproteinase-9 in ovarian epithelial cancer and relation to patient deaths. Ann Diagn Pathol 14: 387-395, 2010.

19. Wakui M, Yokoyama Y, Wang H, Shigeto T, Futagami M and Mizunuma H: Efficacy of a methyl ester of 5-aminolevulinic acid in photodynamic therapy for ovarian cancers. J Cancer Res Clin Oncol, 136: 1143-1150, 2010.

20. Ismail E, Al-Mulla F, Tsuchida S, et al: Carbonyl reductase: a novel metastasis-modulating function. Cancer Res 60: 1173-1176, 2000

21. Cano A, Pérez-Moreno MA, Rodrigo I, et al: The transcription factor snail controls epithelial-mesenchymal transitions by repressing E-cadherin expression. Nat Cell Biol 2: 76-83, 2000.

22. Li LN, Zhou X, Gu Y and Yan J: Prognostic value of MMP-9 in ovarian cancer: a meta-analysis. Asian Pac J Cancer Prev 14: 4107-4113, 2013.

23. Ozalp S, Tanir HM, Yalcin OT, Kabukcuoglu S, Oner U and Uray M: Prognostic value of matrix metalloproteinase- 9 (gelatinase-B) expression in epithelial ovarian tumors. Eur J Gynaecol Oncol 24: 417-420, 2003.

24. Sillanpää S, Anttila M, Voutilainen K, et al: Prognostic significance of matrix metalloproteinase-9 (MMP-9) in epithelial ovarian cancer. Gynecol Oncol 104: 296-303, 2007. 
25. Cowden Dahl KD, Symowicz J, Ning Y, et al: Matrix metalloproteinase 9 is a mediator of epidermal growth factor-dependent e-cadherin loss in ovarian carcinoma cells Cancer Res 68: 4606-4613, 2008.

26. Yokoyama Y, Charnock-Jones DS, Licence D, et al: Vascular endothelial growth factor-D is an independent prognostic factor in epithelial ovarian carcinoma. Br J Cancer 88: 237-244, 2003.

27. Tammela $\mathrm{T}$ and Alitalo K: Lymphangiogenesis: molecular mechanisms and future promise. Cell 140: 460-476, 2010.

28. Abbasi MM, Monfaredan A, Hamishehkar H, Seidi K and Jahanban-Esfahlan R: Novel DOX-MTX nanoparticles improve oral SCC clinical outcome by down regulation of lymph dissemination factor VEGF-C expression in vivo: oral and IV modalities. Asian Pac J Cancer Prev 15: 6227-6232, 2014.
29. Ikeda K, Oki E, Saeki H, et al: Intratumoral lymphangiogenesis and prognostic significance of VEGFC expression in gastric cancer. Anticancer Res 34: 3911-3915, 2014.

30. Gyftopoulos K, Lilis I, Kourea H and Papadaki H: The expression of vascular endothelial growth factor-C correlates with lymphatic microvessel density and lymph node metastasis in prostate carcinoma: an immunohistochemical study. Urol Ann 6: 224-230, 2014.

31. Peppicelli S, Bianchini F and Calorini L: Inflammatory cytokines induce vascular endothelial growth factor- $C$ expression in melanoma-associated macrophages and stimulate melanoma lymph node metastasis. Oncol Lett 8: 1133-1138, 2014. 